\title{
Extracts of Glycyrrhiza uralensis and Isoliquiritigenin Counteract Amyloid- $\beta$ Toxicity in Caenorhabditis elegans
}

Authors

Affiliations
Pille Link ${ }^{1}$, Bernhard Wetterauer ${ }^{1}$, Yujie Fu ${ }^{2}$, Michael Wink ${ }^{1}$

${ }^{1}$ Institute of Pharmacy and Molecular Biotechnology, Heidelberg University, Heidelberg, Germany

${ }^{2}$ Key Laboratory of Forest Plant Ecology, Ministry of Education, Northeast Forestry University, Harbin, China
Key words

- Glycyrrhiza uralensis

- Fabaceae

- LC-MS/MS analysis

- isoliquiritigenin

- amyloid- $\beta$

- Caenorhabditis elegans

received Nov. 4, 2014

revised January 26, 2015

accepted January 29, 2015

\section{Bibliography}

DOI http://dx.doi.org/

10.1055/s-0035-1545724

Published online March 17,

2015

Planta Med 2015; 81: 357-362

(c) Georg Thieme Verlag KG

Stuttgart · New York ·

ISSN 0032-0943

Correspondence

\section{Pille Link}

Institute of Pharmacy and

Molecular Biotechnology

Heidelberg University

Department of Biology

Im Neuenheimer Feld 364

69120 Heidelberg

Germany

Link@uni-heidelberg.de

Correspondence

Prof. Dr. Michael Wink

Institute of Pharmacy and

Molecular Biotechnology

Heidelberg University

Department of Biology

Im Neuenheimer Feld 364

69120 Heidelberg

Germany

Wink@uni-heidelberg.de

\section{Abstract}

\section{$\nabla$}

Alzheimer's disease is a rising threat for modern societies as more and more people reach old age. To date, there is no effective treatment for this condition. In this study, we investigated the potential of Glycyrrhiza uralensis to counteract amyloid- $\beta$ toxicity, one of the key features of Alzheimer's disease. An LC-MS/MS analysis revealed glycyrrhizic acid and glycosylated forms of isoliquiritigenin and liquiritigenin as major constituents of water and methanol extracts of G. uralensis. These extracts and the pure compounds were tested for their activity in two Caenorhabditis elegans models of amyloid- $\beta$ aggregation and amyloid- $\beta$ toxicity, respectively. The number of amyloid- $\beta$ aggregates decreased by $30 \%$ after treatment with isoliquiritigenin, the methanol extract could reduce the number by $14 \%$, liquiritigenin and glycyrrhizic acid by $15 \%$, and the aglycon of glycyrrhizic acid, glycyrrhetinic acid, by $20 \%$. Both extracts and isoliquiritigenin also showed significant activity against acute amyloid- $\beta$ toxicity in

\section{Introduction}

$\nabla$

AD occurs with a prevalence of $5 \%$ in people over 60 and the risk increases with age. About 36 million people were estimated to suffer from this disease in 2010 according to Alzheimer's disease International [1]. Despite these facts, there is still no effective cure for this disease today. Therefore, it is important to continue research for new possible disease modulating substances. Rich sources for potential therapeutic compounds are medicinal plants and their secondary metabolites, which have evolved as a means of protection against herbivores and microbes for plants producing them [2].

Glycyrrhiza species (eng. licorice; Fabaceae) have been known as medicinal plants and sweets for transgenic $C$. elegans that express human amyloid- $\beta$ peptides, delaying the paralysis in this model by $1.8 \mathrm{~h}$ and $1.1 \mathrm{~h}$, respectively. We conclude that secondary compounds of $G$. uralensis may become interesting drug candidates for the treatment of Alzheimer's disease, which, however, need further analysis in other model systems.

$\begin{array}{ll}\text { Abbreviations } \\ \text { A } \beta \text { : } & \text { amyloid-beta } \\ \text { AD: } & \text { Alzheimer's disease } \\ \text { EGCG: } & (-) \text {-epigallocathechin gallate } \\ \text { GA: } & \text { glycyrrhizic acid } \\ \text { GUE: } & \text { Glycyrrhiza uralensis extract } \\ \text { GRA: } & \text { glycyrrhetinic acid } \\ \text { ILG: } & \text { isoliquiritigenin } \\ \text { LG: } & \text { liquiritigenin } \\ \text { NGM: } & \text { nematode growth medium } \\ \text { NMDA: } & \text { N-methyl-D-aspartate } \\ \text { PT } 50: & \text { median paralysis time } \\ \text { TCM: } & \text { traditional Chinese medicine }\end{array}$

centuries both in Europe and Asia. The traditional uses include conditions of the respiratory system and the gastrointestinal tract [3]. Licorice is also used in many formulations of TCM and is the most frequently used herb in the Chinese Formulae Database [4]. Several studies indicate that Glycyrrhi$z a$ can be beneficial in the treatment of AD [5-7]. The most abundant secondary metabolite found in Glycyrrhiza species is the triterpene saponin GA. GA and its aglycone GRA have been shown to have anti-inflammatory and neuroprotective effects $[8,9]$. Next to the triterpenes, the plant also produces many flavonoids. These polyphenols can interact with biomolecules via their phenolic hydroxyl groups and thereby modify the function of many proteins. The flavonoid ILG shows antiinflammatory [10-12] and neuroprotective ef- 


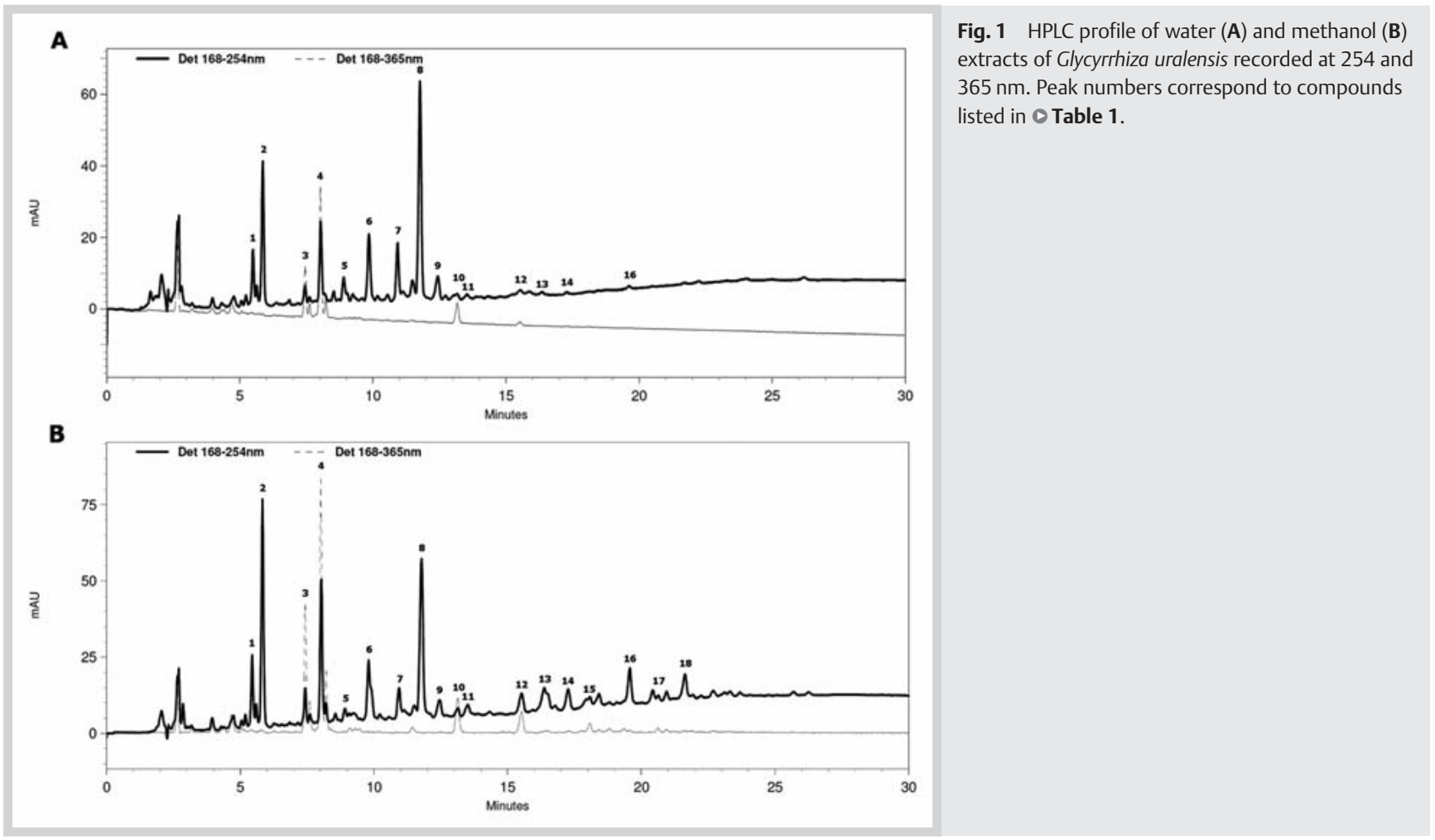

fects $[13,14]$. It is an NMDA receptor antagonist [15]. Additionally, ILG and its isomer LG exhibit antidepressant properties in mice [16] and both have been shown to inhibit neurotoxicity caused by $A \beta$ in rat neurons $[17,18]$.

$\mathrm{A} \beta$ is one of the key proteins in $\mathrm{AD}$ and a potential drug target [19]. This peptide of 38-43 amino acids can take different conformations, build aggregates, and interact with cellular processes. Monomeric $A \beta$ is not very stable in aqueous solutions and builds oligomers, which are toxic to cells [20]. Further aggregation leads to so-called senile plaques that are abundant in the brains of $\mathrm{AD}$ patients.

To better understand $A \beta$ aggregation and its toxic effect in vivo, transgenic Caenorhabditis elegans models have been developed [21]. These worms express the human $A \beta_{3-42}$ peptide in their muscles, where it aggregates and forms plaques. The toxicity to the surrounding muscle cells manifests in a paralysis phenotype. This in vivo model can assess the bioavailability of the compounds in contrast to in vitro aggregation experiments or toxicity assays with cell cultures. In the present study, the effect of Glycyrrhiza uralensis Fisch. ex DC and its secondary metabolites on $\mathrm{A} \beta$ aggregation and acute $A \beta$ toxicity were studied using transgenic C. elegans strains.

\section{Results}

$\nabla$

The HPLC analysis revealed several compounds ( Fig. 1), 18 of which were further analyzed ( Table 1). Substances 1-9, 11, 12,15 , and 18 could be identified according to their mass spectra and to published data for GUEs [22-24]. GA and glycosylated forms of LG and ILG are among the most abundant compounds in this extract. In the water extract ( $\bullet$ Fig. $1 \mathrm{~A}$ ), fewer compounds were found. The more lipophilic substances 15, 17, and 18 were missing; 12,14 , and 16 were only present in traces. Also, the oth- er compounds were less concentrated in the water extract, except for the saponins $\mathbf{7}$ and $\mathbf{8}$ that have a slightly higher abundance in the water extract compared to the methanol extract.

CL2006, a transgenic $C$. elegans strain that constitutively expresses the human $A \beta$ peptide, was used to test the effect on $A \beta$ aggregation in vivo. $A \beta$ aggregates can be visualized by thioflavin $\mathrm{S}$ staining. Fig. 2 shows typical pictures for the thioflavin $\mathrm{S}$ staining of a control (A) and a GUE-treated worm (B). GUE $(500 \mu \mathrm{g} / \mathrm{mL})$ and the pure substances GA, GRA, LG, and ILG $(50 \mu \mathrm{g} / \mathrm{mL})$ all significantly reduced the number of $A \beta$ aggregates ( Fig. 2C). The effect of ILG (30\% reduction) was similar to the positive control EGCG (a polyphenol from green tea) $(100 \mu \mathrm{g} /$ $\mathrm{mL}$ ) that reduced the number of $A \beta$ aggregates by $35 \%$. GUE, GA, GRA, and LG had a weaker effect (a reduction by $14 \%$ for GUE, $15 \%$ for GA and LG, and $20 \%$ for GRA). For all treatments, the low concentrations had no significant effect; a significant effect was reached at a concentration of $50 \mu \mathrm{g} / \mathrm{mL}$ for GRA, LG, and ILG and at a concentration of $500 \mu \mathrm{g} / \mathrm{mL}$ for GUE, as can be seen on - Fig. 2D. Therefore, the treatments can be considered dose-dependent.

The paralysis assay with strain CL4176 reveals the effect of the treatment on $A \beta$ toxicity. Since the methanol that was used as a solvent for methanol GUE, ILG, LG, GRA, and GA had a paralysis delaying effect itself, the results of the compounds were compared to a methanol-treated control (० Fig.3A,B). EGCG and water GUE that were solved in water were compared to a watertreated control ( $\odot$ Fig. $3 \mathrm{C}$ ). EGCG was used as a positive control in a concentration of $100 \mu \mathrm{g} / \mathrm{mL}$. It increased the median time to paralysis $\left(\mathrm{PT}_{50}\right)$ by $2.7 \mathrm{~h}$ ( Table 2 ). Treatments with $500 \mu \mathrm{g} / \mathrm{mL}$ methanol GUE, $200 \mu \mathrm{g} / \mathrm{mL}$ GUE (both methanol and water), and $50 \mu \mathrm{g} / \mathrm{mL}$ ILG could also significantly increase the time until paralysis compared to the control. The water extract was less effective than the positive control, but with a $1.7 \mathrm{~h}$ increase in $\mathrm{PT}_{50}$, it was more effective than the same amount of methanol extract. 


\begin{tabular}{|c|c|c|c|c|c|c|}
\hline Nr. & RT in min & $\lambda_{\max }$ & {$[\mathrm{M}-\mathrm{H}]^{-}$} & Daughter ions & Tentative identification & \multirow{19}{*}{$\begin{array}{l}\text { Table } 1 \text { Retention times, absorp- } \\
\text { tion maxima, and results of the } \\
\text { MS/MS analysis of } G \text {. uralensis ex- } \\
\text { tract. }\end{array}$} \\
\hline 1 & 5.4 & $198,214,277,310$ & 549 & 255,429 & liquiritin apioside & \\
\hline 2 & 5.8 & $199,215,277,311$ & 417 & 255 & liquiritin & \\
\hline 3 & 7.4 & 206,362 & 549 & 225,417 & isoliquiritin apioside & \\
\hline 4 & 8.0 & 202,361 & 417 & 255 & isoliquiritin & \\
\hline 5 & 8.9 & 214 & 983 & $821,803,351$ & licorice saponin $\mathrm{A} 3$ & \\
\hline 6 & 9.8 & $199,215,276,311$ & 879 & $351,861,703,817$ & 22-acetoxyglycyrrhizin & \\
\hline 7 & 10.9 & 215 & 837 & $351,661,819,775$ & licorice saponin G2 & \\
\hline 8 & 11.8 & 218,250 & 821 & $351,803,645,759$ & glycyrrhizic acid & \\
\hline 9 & 12.4 & 217 & 821 & $351,803,645,759$ & licorice saponin $\mathrm{H} 2$ & \\
\hline 10 & 13.1 & 218,370 & 821 & $351,510,645,777$ & & \\
\hline 11 & 13.5 & 218 & 823 & $351,805,647$ & licorice saponin 12 & \\
\hline 12 & 15.5 & 218,348 & 367 & 298,337 & glycycoumarin & \\
\hline 13 & 16.3 & 219 & 353 & 298,284 & & \\
\hline 14 & 17.3 & 221,287 & 353 & 297,285 & & \\
\hline $15^{*}$ & 18.1 & 221,347 & 365 & $307,295,350$ & glycyrol & \\
\hline 16 & 19.6 & 221 & 351 & $283,265,307$ & & \\
\hline $17^{*}$ & 20.6 & 219, 292 & 423 & 229,193 & & \\
\hline $18^{*}$ & 21.6 & 222,268 & 421 & 284 & & \\
\hline
\end{tabular}

* Only present in the methanol extract

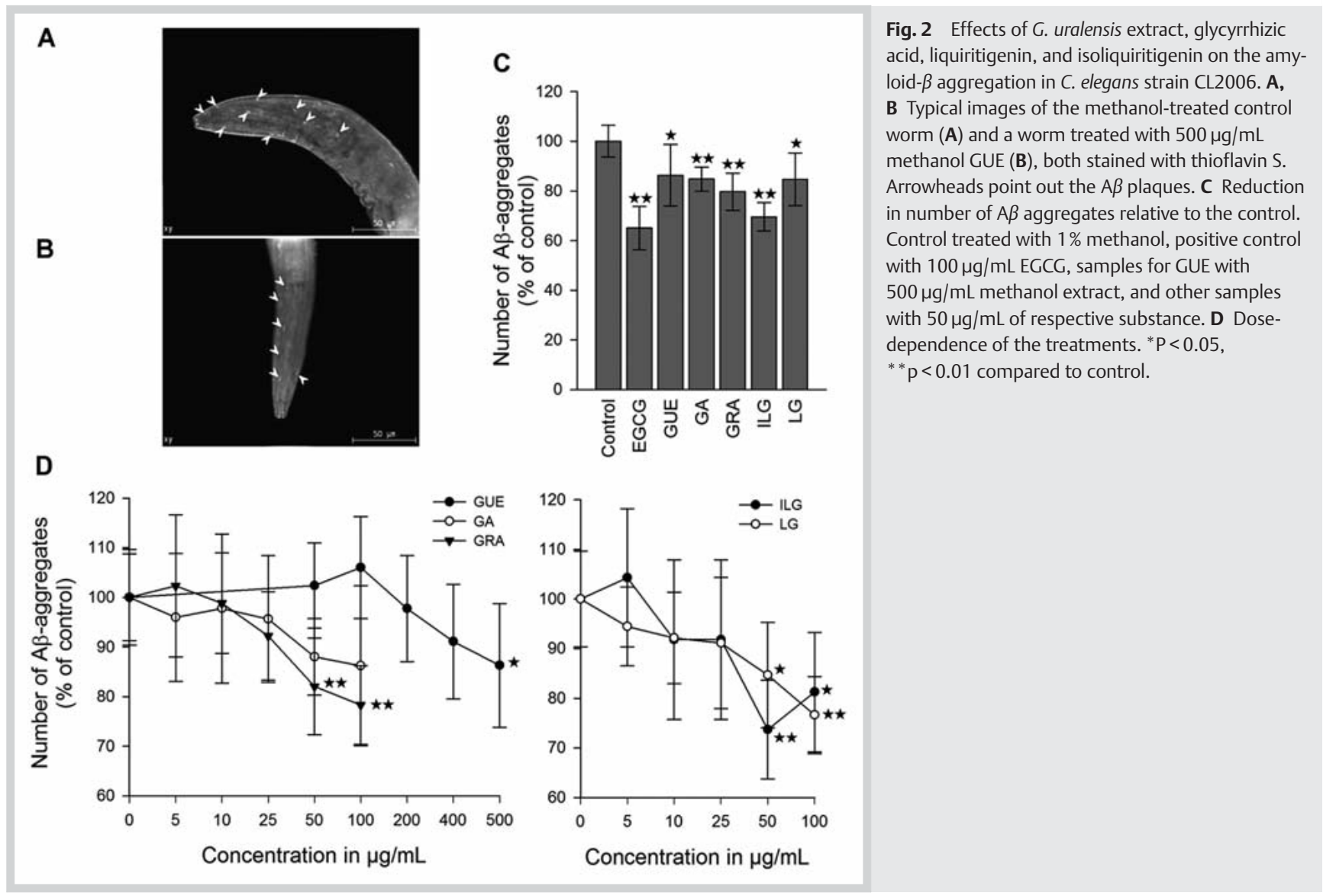

Treatments with LG, GRA, and GA showed no significant increase. The control strain CL802 showed no paralysis when treated with extracts or pure compounds.

\section{Discussion}

\section{$\nabla$}

All the tested substances had a significant effect on $A \beta$ aggregation and lowered the number of plaques in the $C$. elegans strain CL2006, but the effects were much weaker in the test for acute $\mathrm{A} \beta$ toxicity. Only GUE and ILG could delay the paralysis in CL4176. In this $C$. elegans strain, amyloid toxicity was observed despite the small number of amyloid deposits, suggesting that 


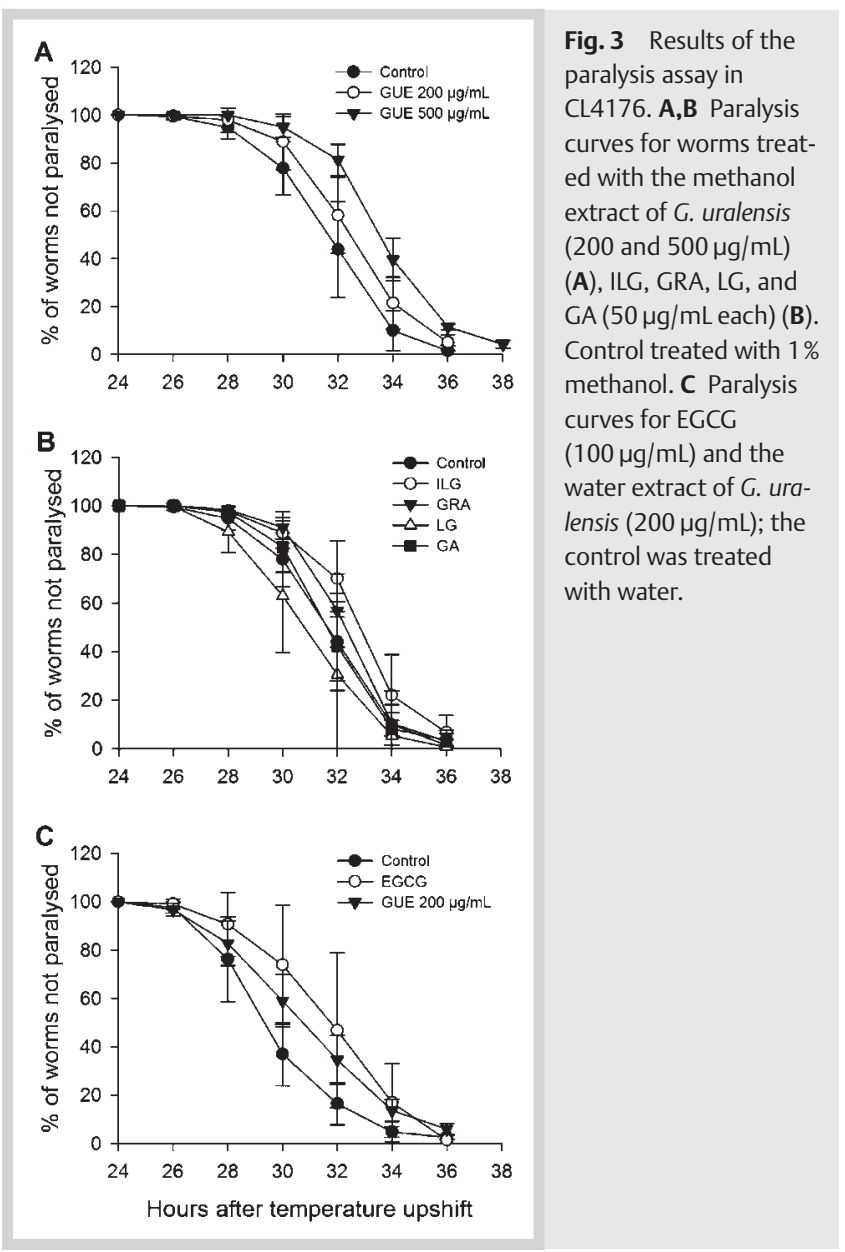

the toxicity was exerted by $A \beta$ oligomers [25]. Possibly, the other tested substances are only able to destabilize fibrillar $A \beta$, resulting in a decreased number of plaques, but they fail to interact with the toxic oligomers. To confirm the interactions with different forms of $A \beta$, further studies have to be performed.

Along with a possible direct interaction of GUE and its constituents with $A \beta$, the effects seen can also be explained by the supporting nature of the drugs on the innate defensive mechanisms of the worms. Cohen et al. have proposed that there are two possibilities in $C$. elegans to deal with toxic $A \beta$ oligomers on a molecular level: deposition in less toxic plaques or disaggregation and degradation [26]. Based on this hypothesis, the observation that GUE and ILG reduce both toxicity and the number of $A \beta$ aggregates can be interpreted as an enhancement of the latter pathway involving HSF-1. This transcription factor is highly conserved and has an ortholog in humans. Since clearance of $A \beta$ is decreased in patients with Alzheimer's disease [27], a stimulation of the degradation is a promising therapeutic strategy, and the results shown here suggest that GUE or ILG might be potential drugs.

The results for ILG are in accordance with earlier results in cell cultures [18]. Interestingly, the isomer LG fails to delay the paralysis in CL4176, although it could prevent apoptosis of cultured rat neurons at even lower concentrations than ILG [17]. The suggested mechanism of action for LG involves the estrogen receptor $\beta$ and Notch-2 signaling, resulting in lower astrogliosis [28]. Although C. elegans expresses homologues of an estrogen receptor and Notch-2, the nervous system of $C$. elegans is much simpler. For example, it does not contain astrocytes. Therefore, the
Table 2 Delay of paralysis in C. elegans strain CL4176.

\begin{tabular}{|c|c|c|}
\hline Treatment & $\mathrm{PT}_{50} \pm \mathrm{SD}$ & Significance \\
\hline $1 \%$ methanol & $33.7 \pm 0.6$ & \\
\hline $200 \mu \mathrm{g} / \mathrm{mL}$ GUE & $34.4 \pm 0.7$ & $p<0.05$ \\
\hline $500 \mu \mathrm{g} / \mathrm{mL}$ GUE & $35.5 \pm 0.4$ & $p<0.01$ \\
\hline $50 \mu \mathrm{g} / \mathrm{mL}$ GA & $33.7 \pm 0.6$ & \\
\hline $50 \mu \mathrm{g} / \mathrm{mL}$ GRA & $34.3 \pm 0.7$ & \\
\hline $50 \mu \mathrm{g} / \mathrm{mL} \mathrm{LG}$ & $32.8 \pm 1.5$ & \\
\hline $50 \mu \mathrm{g} / \mathrm{mL}$ ILG & $34.8 \pm 0.7$ & $p<0.05$ \\
\hline Water & $30.9 \pm 0.8$ & \\
\hline $100 \mu \mathrm{g} / \mathrm{mL}$ EGCG & $33.6 \pm 1.7$ & $p<0.01$ \\
\hline $200 \mu \mathrm{g} / \mathrm{mL}$ GUE & $32.7 \pm 0.8$ & $p<0.05$ \\
\hline
\end{tabular}

$\mathrm{PT}_{50}$ values in hours after temperature upshift, calculated from paralysis curves ( $\odot$ Fig. 3); p values compared to the respective control (methanol or water)

inability of this study to reproduce the positive effects of LG seen in mice and cell cultures might be due to lack of a corresponding target or pathway in the chosen model organism.

The beneficial effect of ILG against $A \beta$ toxicity might be achieved by NMDA receptor antagonism, antioxidative, or antiinflammatory properties of this compound. The reports about antioxidant properties of ILG are somewhat inconsistent. ILG has poor radical scavenging activity compared to other phenolics in licorice, but it can prevent LDL oxidation [29] and has shown beneficial effects in in vivo experiments [30]. Thus, the antioxidant properties in the given model system should be determined before any conclusions about the involvement of this mechanism can be made. It has also been shown that ILG can inhibit $A \beta$ aggregation [31]. Possibly, all these effects contribute to the observed results, but the exact mechanism of action needs further elucidation.

ILG was only found in traces in GUE, but its glycosylated forms isoliquiritin and isoliquiritin apioside were far more abundant. Suggesting that the effect of GUE is based on ILG, it is possible that the glycosylated forms found in the extract have similar effects as ILG or are cleaved either by the Escherichia coli used as a food source for $C$. elegans or by the enzymes of $C$. elegans itself. Still there is the possibility that this compound alone is not responsible for the effect observed by the treatment with GUE. Therefore, some other not yet tested substances in this extract might either have a toxicity ameliorating effect against $A \beta$ themselves or can synergistically contribute to the effect of ILG.

The observation that the water extract had an even stronger effect than the methanol extract against the paralysis supports the suggestion that ILG is not the only active component of the extracts. The water extract contains less derivatives of ILG, but equal or slightly higher amounts of saponins. Saponins can enhance the effect of a drug by making the cell membranes more permeable and allowing the active substances to reach their intracellular targets. On the other hand, they can be active themselves, although GA, the only saponin tested here, could not counteract the $A \beta$ toxicity alone. The possible synergistic effects of Glycyrrhiza saponins need to be further investigated.

The effects of GUE and ILG were quite low given the relatively high drug concentrations, which may be due to an incomplete absorption of the drugs into the body of $C$. elegans. Studies in mice and rats have shown a substantial absorbance for ILG, the major active component of GUE in current experiments, however, the bioavailability in these studies accounted only for $22-34 \%$ $[32,33]$. According to Zheng et al., the amount of drugs absorbed by C. elegans is similar to that found in mice [34], and therefore 
the low bioavailability may also account for the low effects observed in the present study. It is also possible that part of the drugs were metabolized by the bacteria used as a food source, thereby lowering the effective drug concentration in the experiments. For further studies in C. elegans, it is therefore recommended to use dead bacteria.

In conclusion, it can be stated that ILG and GUE exhibit significant effects, counteracting the pathological effects of $A \beta$ in C. elegans. While this nematode is a good tool for identifying new drug candidates and studying specific targets, it lacks the complexity of the vertebrate body. Therefore, ILG and possibly other constituents of $G$. uralensis should be further studied as possible drug candidates against $\mathrm{AD}$ in vertebrate systems.

\section{Materials and Methods}

$\nabla$

Chemicals

EGCG (from green tea, purity $\geq 95 \%$ ) and thioflavin S were purchased from Sigma-Aldrich Co. ILG (purity >99\%), GA (purity $75 \%$ ), and glycyrrhetinic acid (GRA) (purity > 96\%) were isolated by Prof. Dr. Yujie Fu. LG (purity $>98 \%$ ) was a kind gift from Dr. Qiujun Lu, Wangjing Science and Technology Park. The purity of ILG, GA, GRA, and LG was confirmed by HPLC as described in [35].

\section{Plant material}

Dried roots of G. uralensis were purchased in China and provided to our Institute by Prof. Dr. Thomas Efferth. The authenticity of the plant material was confirmed by DNA barcoding of the ITS sequence (GenBank accession number KM588200). Voucher specimens with the registration number $\mathrm{P} 6873$ are deposited at the Department of Biology, Institute of Pharmacy and Molecular Biotechnology, Heidelberg University, Germany.

Fifty $g$ of the dried plant material were pulverized and extracted with $300 \mathrm{ml}$ of water or methanol at moderate heat for $4 \mathrm{~h}$. The water extract was lyophilized (DER 13.8:1), the methanol extract was reduced in a rotary evaporator to dryness (DER $11.1: 1$ ), and both extracts were stored at $-20^{\circ} \mathrm{C}$.

\section{LC-MS/MS analysis of the extract}

The composition of the methanol extract was analyzed on an LCQ-Duo ion trap mass spectrometer with an ESI source (ThermoQuest) coupled to a Beckman Gold HPLC system (solvent module 125P, PDA detector 168) with a LiChroCART RP18 column ( $5 \mu \mathrm{m}, 250 \times 4 \mathrm{~mm}$, Merck). A gradient of water and acetonitrile with $0.1 \%$ formic acid each was applied from $20 \%$ to $80 \%$ ACN in $20 \mathrm{~min}$ and isocratic for $10 \mathrm{~min}$ with the latter conditions. The flow rate was $1 \mathrm{~mL} / \mathrm{min}$ throughout the whole run. The absorption maxima were determined in background-subtracted spectra by 32 Karat $^{\mathrm{TM}}$ software (Beckman Coulter, Inc.). The MS operated in the negative mode with a capillary voltage of $-10 \mathrm{~V}$, a source temperature of $200^{\circ} \mathrm{C}$, and high purity nitrogen as a sheath and auxiliary gas at a flow rate of 80 and 40 (arbitrary units), respectively. The ions were detected in a mass range of $50-2000 \mathrm{~m} / \mathrm{z}$. Major peaks were identified by comparison with published data [22-24].

\section{Caenorhabditis elegans strains and culturing conditions}

The $C$. elegans strains CL2006 (constitutively expressing A $\beta$ in its muscle cells) [36], CL4176 (a temperature inducible strain expressing $A \beta$ in its muscle cells) [25], and CL802 (standard control for CL4176) were obtained from Caenorhabditis Genetics Center.
The worms were kept on NGM at $20^{\circ} \mathrm{C}$ (CL2006) or $16^{\circ} \mathrm{C}$ (CL4176 and CL802) and fed with $E$. coli OP50. For all experiments, C. elegans eggs, gained from gravid hermaphrodites by sodium hypochlorite treatment, were used to obtain an age-synchronized population. The $A \beta$ aggregation assay was conducted in S-medium with E. coli OP50 $\left(1 \times 10^{9}\right.$ cells $\left./ \mathrm{mL}\right)$ as a food source [37].

\section{Amyloid- $\beta$ aggregation in Caenorhabditis elegans CL2006} The worms were treated with $500 \mu \mathrm{g} / \mathrm{mL}$ methanol extract of $G$. uralensis or $50 \mu \mathrm{g} / \mathrm{mL}$ pure substances (solved in methanol) on day two after hatching. The concentrations were chosen based on a dose-dependence experiment, where various concentrations between $5-100 \mu \mathrm{g} / \mathrm{mL}$ for pure compounds and 50 $500 \mu \mathrm{g} / \mathrm{mL}$ for the extract were tested. A treatment with $100 \mu \mathrm{g} /$ mL EGCG served asa positive control and the solvent methanol ( $1 \%$ of the final volume) as a negative control. On day six, the worms were fixed, followed by thioflavin $S$ staining of the $A \beta$ aggregates as described before $[38,39]$. In contrast to the original method, $0.013 \%$ of thioflavin $\mathrm{S}$ in $50 \%$ ethanol was used. The $\mathrm{A} \beta$ aggregates in the head region of the worms were quantified using Nikon Eclipse Ni-E with an FITC filter and 40× objective. Pictures were acquired with a DS-Qi1Mc camera, and deconvolution was performed with Huygens software by SVI.

\section{Paralysis assay in Caenorhabditis elegans CL4176}

The paralysis assay was conducted as described by Dostal and Link [40]. The methanol extract of G. uralensis $(200 \mu \mathrm{g} / \mathrm{mL}$ and $500 \mu \mathrm{g} / \mathrm{mL})$, the water extract of $G$. uralensis $(200 \mu \mathrm{g} / \mathrm{mL})$, or one of the pure substances, ILG, LG, GA, or GRA ( $50 \mu \mathrm{g} / \mathrm{mL})$, was added to each NGM plate. EGCG $(100 \mu \mathrm{g} / \mathrm{mL})$ and the respective solvent (1\%) were used as controls. The worms were kept at $16^{\circ} \mathrm{C}$ for $36 \mathrm{~h}$, and then the temperature was upshifted to $25^{\circ} \mathrm{C}$ to induce the transgene expression. Paralysis was scored every $2 \mathrm{~h}$ for $12 \mathrm{~h}$ or, in the case of GUE $500 \mu \mathrm{g} / \mathrm{mL}$, for $14 \mathrm{~h}$ starting at $24 \mathrm{~h}$ after the temperature upshift. Worms who failed to move as a response to a touch with a platin wire were counted as paralyzed.

\section{Statistical analysis}

Data are expressed as the mean \pm SD of at least three independent experiments ( $n=4$ for the $A \beta$ aggregation experiments, $n=3$ for the paralysis assay). With the data from the paralysis assays, a survival analysis using the life table method was conducted to calculate the median paralysis times $\left(\mathrm{PT}_{50}\right)$. Differences were analyzed in StatView software using ANOVA and Dunnet's test with $\mathrm{p}<0.05$ as the threshold for significance.

\section{Acknowledgements \\ $\nabla$}

Instruments and software for microscopic evaluation were provided by Nikon Imaging Center at Heidelberg University. Worm strains used in the present study were provided by Caenorhabditis Genetics Center at the University of Minnesota, funded by the NIH National Center for Research Resources (NCRR). We are also grateful to Prof. Dr. Thomas Efferth (University of Mainz) for providing the plant material and to Dr. Qiujun Lu (Wangjing Science and Technology Park) for providing the pure substance liquiritigenin. 


\section{Conflict of Interest}

$\nabla$

The authors declare no conflict of interest.

\section{References}

1 Prince M, Jackson J, editors. World Alzheimer Report. London: Alzheimer's Disease International; 2009

2 Wink M. Evolution of secondary metabolites from an ecological and molecular phylogenetic perspective. Phytochemistry 2003; 64: 3-19

3 Asl MN, Hosseinzadeh H. Review of pharmacological effects of Glycyrrhiza sp. and its bioactive compounds. Phytother Res 2008; 22: 709724

4 Guo J, Shang E, Zhao J, Fan X, Duan J, Qian D, Tao W, Tang Y. Data mining and frequency analysis for licorice as a "Two-Face" herb in Chinese Formulae based on Chinese Formulae Database. Phytomedicine 2014; 21 : 1281-1286

5 Dhingra D, Parle M, Kulkarni SK. Memory enhancing activity of Glycyrrhiza glabra in mice. J Ethnopharmacol 2004; 91: 361-365

6 Kanno H, Kawakami Z, Iizuka S, Tabuchi M, Mizoguchi K, Ikarashi Y, Kase Y. Glycyrrhiza and Uncaria Hook contribute to protective effect of traditional Japanese medicine yokukansan against amyloid beta oligomer-induced neuronal death. J Ethnopharmacol 2013; 149: 360-370

7 Ahn J, Um M, Choi W, Kim S, Ha T. Protective effects of Glycyrrhiza uralensis Fisch. on the cognitive deficits caused by beta-amyloid peptide 25-35 in young mice. Biogerontology 2006; 7: 239-247

8 Wang CY, Kao TC, Lo WH, Yen GC. Glycyrrhizic acid and 18beta-glycyrrhetinic acid modulate lipopolysaccharide-induced inflammatory response by suppression of NF-kappaB through PI3K p110delta and p 110gamma inhibitions. J Agric Food Chem 2011; 59: 7726-7733

9 Luo L, Jin Y, Kim ID, Lee JK. Glycyrrhizin attenuates kainic acid-induced neuronal cell death in the mouse hippocampus. Exp Neurobiol 2013; 22: $107-115$

10 Kim JY, Park SJ, Yun KJ, Cho YW, Park HJ, Lee KT. Isoliquiritigenin isolated from the roots of Glycyrrhiza uralensis inhibits LPS-induced iNOS and COX-2 expression via the attenuation of NF-kappaB in RAW 264.7 macrophages. Eur J Pharmacol 2008; 584: 175-184

11 Takahashi T, Takasuka N, Iigo M, Baba M, Nishino H, Tsuda H, Okuyama $T$. Isoliquiritigenin, a flavonoid from licorice, reduces prostaglandin E2 and nitric oxide, causes apoptosis, and suppresses aberrant crypt foci development. Cancer Sci 2004; 95: 448-453

12 Park SJ, Youn HS. Suppression of homodimerization of toll-like receptor 4 by isoliquiritigenin. Phytochemistry 2010; $71: 1736-1740$

13 Hwang CK, Chun HS. Isoliquiritigenin isolated from licorice Glycyrrhiza uralensis prevents 6-hydroxydopamine-induced apoptosis in dopaminergic neurons. Biosci Biotechnol Biochem 2012; 76: 536-543

14 Yang EJ, Min JS, Ku HY, Choi HS, Park MK, Kim MK, Song KS, Lee DS. Isoliquiritigenin isolated from Glycyrrhiza uralensis protects neuronal cells against glutamate-induced mitochondrial dysfunction. Biochem Biophys Res Commun 2012; 421: 658-664

15 Kawakami Z, Ikarashi $Y$, Kase $Y$. Isoliquiritigenin is a novel NMDA receptor antagonist in kampo medicine yokukansan. Cell Mol Neurobiol 2011; 31: 1203-1212

16 Wang W, Hu X, Zhao Z, Liu P, Hu Y, Zhou J, Zhou D, Wang Z, Guo D, Guo H. Antidepressant-like effects of liquiritin and isoliquiritin from Glycyrrhiza uralensis in the forced swimming test and tail suspension test in mice. Prog Neuropsychopharmacol Biol Psychiatry 2008; 32: 11791184

17 Liu RT, Zou LB, Lu OJ. Liquiritigenin inhibits Abeta(25-35)-induced neurotoxicity and secretion of Abeta(1-40) in rat hippocampal neurons. Acta Pharmacol Sin 2009; 30: 899-906

18 Lee HK, Yang EJ, Kim JY, Song KS, Seong YH. Inhibitory effects of glycyrrhizae radix and its active component, isoliquiritigenin, on Abeta(2535)-induced neurotoxicity in cultured rat cortical neurons. Arch Pharm Res 2012; 35: 897-904

19 Hardy J, Selkoe DJ. The amyloid hypothesis of Alzheimer's disease: progress and problems on the road to therapeutics. Science 2002; 297: 353-356
20 Walsh DM, Selkoe DJ. A beta oligomers - a decade of discovery. J Neurochem 2007; 101: 1172-1184

21 Link CD. C. elegans models of age-associated neurodegenerative diseases: lessons from transgenic worm models of Alzheimer's disease. Exp Gerontol 2006; 41: 1007-1013

22 Montoro P, Maldini M, Russo M, Postorino S, Piacente S, Pizza C. Metabolic profiling of roots of liquorice (Glycyrrhiza glabra) from different geographical areas by ESI/MS/MS and determination of major metabolites by LC-ESI/MS and LC-ESI/MS/MS. J Pharm Biomed Anal 2011; 54: 535544

23 Farag MA, Porzel A, Wessjohann LA. Comparative metabolite profiling and fingerprinting of medicinal licorice roots using a multiplex approach of GC-MS, LC-MS and 1D NMR techniques. Phytochemistry 2012; 76: 60-72

24 Lee YS, Kim SH, Kim JK, Shin HK, Kang YH, Park JH, Lim SS. Rapid identification and preparative isolation of antioxidant components in licorice. J Sep Sci 2010; 33: 664-671

25 Drake J, Link CD, Butterfield DA. Oxidative stress precedes fibrillar deposition of Alzheimer's disease amyloid beta-peptide (1-42) in a transgenic Caenorhabditis elegans model. Neurobiol Aging 2003; 24: 415420

26 Cohen E, Bieschke J, Perciavalle RM, Kelly JW, Dillin A. Opposing activities protect against age-onset proteotoxicity. Science 2006; 313: $1604-1610$

27 Mawuenyega KG, Sigurdson W, Ovod V, Munsell L, Kasten T, Morris JC, Yarasheski KE, Bateman RJ. Decreased clearance of CNS beta-amyloid in Alzheimer's disease. Science 2010; 330: 1774

28 Liu RT, Tang JT, Zou LB, Fu JY, Lu $Q J$. Liquiritigenin attenuates the learning and memory deficits in an amyloid protein precursor transgenic mouse model and the underlying mechanisms. Eur J Pharmacol 2011; 669: 76-83

29 Vaya J, Belinky PA, Aviram M. Antioxidant constituents from licorice roots: isolation, structure elucidation and antioxidative capacity toward LDL oxidation. Free Radic Biol Med 1997; 23: 302-313

30 Zhang X, Zhu P, Zhang X, Ma Y, Li W, Chen JM, Guo HM, Bucala R, Zhuang $\mathrm{J}$, Li J. Natural antioxidant-isoliquiritigenin ameliorates contractile dysfunction of hypoxic cardiomyocytes via AMPK signaling pathway. Mediators Inflamm 2013; 2013: 390890

31 Chen YP, Zhang ZY, Li YP, Li D, Huang SL, Gu LQ Xu J, Huang ZS. Syntheses and evaluation of novel isoliquiritigenin derivatives as potential dual inhibitors for amyloid-beta aggregation and 5-lipoxygenase. Eur J Med Chem 2013; 66: 22-31

32 Han SY, Chin YW, Choi YH. A new approach for pharmacokinetic studies of natural products: measurement of isoliquiritigenin levels in mice plasma, urine and feces using modified automated dosing/blood sampling system. Biomed Chromatogr 2013; 27: 741-749

33 Qiao H, Zhang X, Wang T, Liang L, Chang W, Xia H. Pharmacokinetics, biodistribution and bioavailability of isoliquiritigenin after intravenous and oral administration. Pharm Biol 2014; 52: 228-236

34 Zheng SQ Ding AJ, Li GP, Wu GS, Luo HR. Drug absorption efficiency in Caenorhabditis elegans delivered by different methods. PLoS One 2013; 8: e56877

35 Zeng L, Zhang RY, Meng T, Lou ZC. Determinatin of nine flavonoids and coumarins in licorice root by high-performance liquid chromatography. J Chromatogr A 1990; 513: 247-254

36 Link CD. Expression of human beta-amyloid peptide in transgenic Caenorhabditis elegans. Proc Natl Acad Sci U S A 1995; 92: 9368-9372

37 Sulston J, Hodgkin J. Methods. In: Wood WB, editor. The nematode Caenorhabditis elegans. Cold Spring Harbor, New York: Cold Spring Harbor Laboratory Press; 1988: 587-606

38 Fay DS, Fluet $A$, Johnson $C$, Link $C D$. In vivo aggregation of $\beta$-amyloid peptide variants. J Neurochem 1998; 71: 1616-1625

39 Abbas S, Wink M. Epigallocatechin gallate inhibits beta amyloid oligomerization in Caenorhabditis elegans and affects the daf-2/insulin-like signaling pathway. Phytomedicine 2010; 17: 902-909

40 Dostal V, Link $C D$. Assaying beta-amyloid toxicity using a transgenic $C$. elegans model. J Vis Exp 2010; 44: 11-16 